\title{
DISEÑO DE ROTOR PARA UN AEROGENERADOR \\ DE BAJAS VELOCIDADES
}

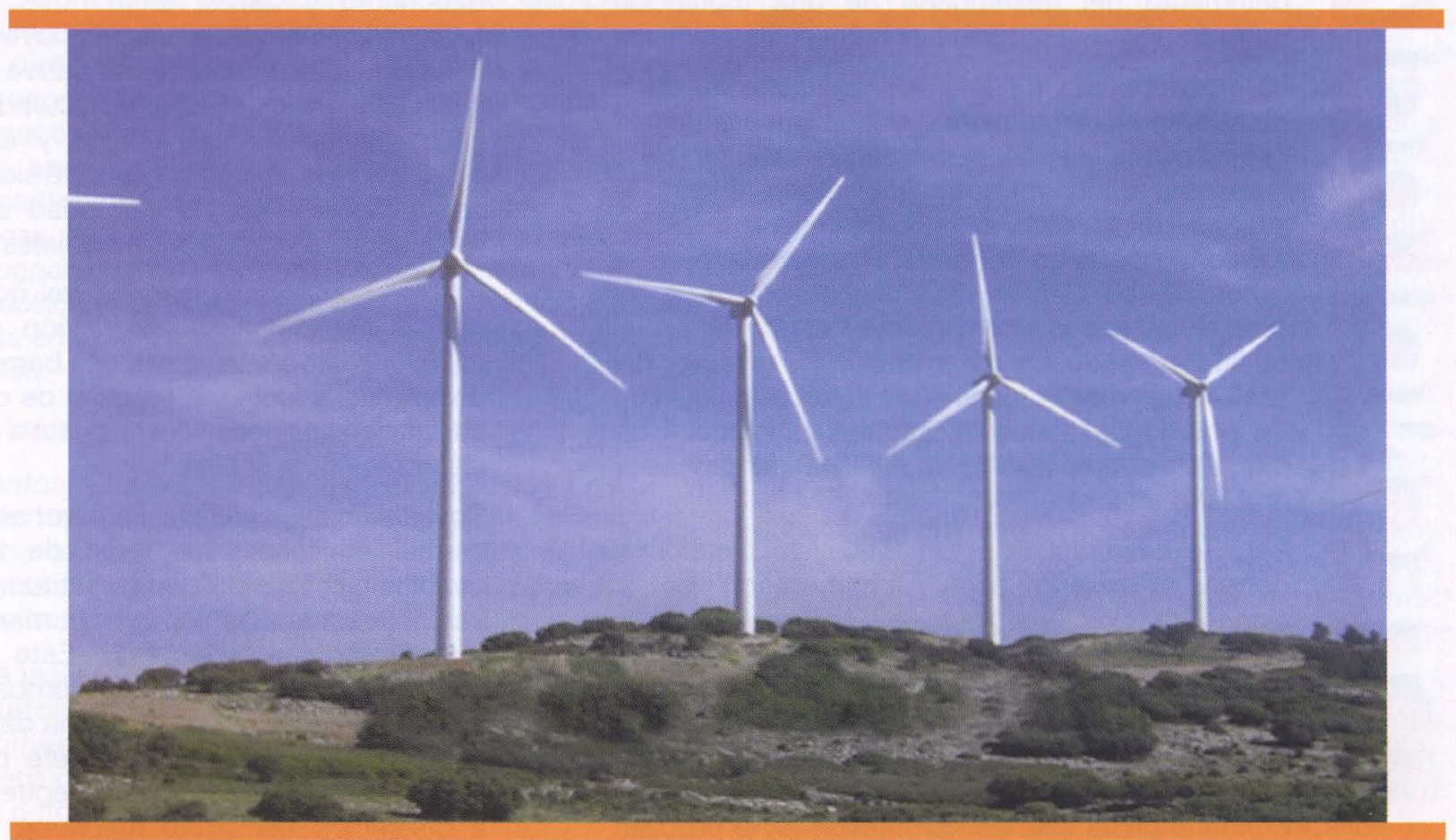

\section{RESUMEN}

En los últimos años ha existido un creciente interés en el diseño y el estudio de turbinas eólicas de baja velocidad debido a la mayor cantidad de lugares donde este potencial eólico esta presente. Usando elementos de la teoría de momentum del álabe (BEM) y la teoría de la circulación, este articulo describe un método basado en el análisis del cambio de circulación y momentum a través de cada estación del álabe, permitiendo cálculos mas precisos y optimizando así el rendimiento del rotor en turbinas eólicas de eje horizontal, baja velocidad de viento y paso fijo. La comparación de los resultados obtenidos con datos provenientes de turbinas eólicas reales muestra una alta similitud.

Palabras claves. Aerogenerador, torque, circulación, estación.

\section{ABSTRACT}

Wind turbine design has become an interesting topic studied around the world. This research presents a method based on the momentum theory and the flow analysis through each blade station. This investigation allows the performance optimization of the main rotor shaft that rotates around the horizontal axis. The results obtained where very similar compared with real wind turbines.

Key words. Aero generator, cogging torque, momentum, flow movement, stations, tip speed ratio, twist.

\section{NOMENCLATURA}

A Factor de reducción de la velocidad axial

AR Aspect ratio

a' Factor de aumento de la velocidad tangencial

An Coeficientes de la función circulación

Cl Coeficiente de Lift

Cd Coeficiente de drag

Da Drag 3d

e Factor de eficiencia de Oswald

I Número de la estación del álabe analizada

I Número total de estaciones

L, I Longitud del álabe

Li Lift 3d

* Ingeniero Aeronáutico Universidad de San Buenaventura Bogotá D.C., Colombia 
U Velocidad absoluta del viento

$\mathrm{Vrl}$ Magnitud de la velocidad relativa

$\alpha \quad$ Ángulo de ataque con respecto a la horizontal $\alpha^{\text {opt }}$ Ángulo de ataque óptimo

\section{$\lceil$ Circulación}

$\lambda \quad$ Taperado

$\phi \quad$ Estación analizada (en radianes), siendo la primera 0 y la última $\mathrm{p} / 2$

$\omega \quad$ Velocidad angular del rotor

\section{INTRODUCCIÓN}

La energía eólica es en la actualidad el tipo de energía alternativa de mayor crecimiento a nivel mundial, sin embargo este desarrollo ha sido enfocado al desarrollo de aerogeneradores para viento de alta velocidad*, por lo cual resulta importante estudiar el diseño de aerogeneradores que funcionen en zonas de vientos de baja velocidad ( 3.5 a $8.5 \mathrm{~m} / \mathrm{s}$ ), incrementando de esta manera el campo de acción de la energía eólica. Para el desarrollo de este proyecto resulta importante establecer los requerimientos de diseño aerodinámico, y establecer los patrones teóricos que deben ser seguidos para de esta forma, determinar un diseño de rotor de aerogenerador que resulte siendo eficiente en los vientos de baja velocidad, y que otorgue mejores prestaciones que otros aerogeneradores existentes, bajo las condiciones de operación dadas.

\section{REQUERIMIENTOS DE DISEÑO}

Con el fin de establecer los parámetros de diseño, resulta importante conocer los requerimientos de diseño respecto a la selección de perfiles, el tamaño del rotor, y las velocidades de operación.

Selección del perfil. Para la selección tuvieron en cuenta los perfiles de la serie Sg[1], diseñados específicamente para pequeñas turbinas de viento, de igual manera se analizaron otros perfiles que se podían adaptar a este propósito. Aquí se estableció un análisis para número de Reynolds dentro de un régimen laminar y se determinó que los efectos por compresibilidad no van influenciar a los diferentes parámetros del análisis aerodinámico debido a que el valor de número de Mach para este caso es menor a $0.5[2]$.

Se buscó que la relación $\mathrm{Cl} / \mathrm{Cd}$ fuese máxima debido a que se tendría mayor sustentación y muy poca resistencia (drag) obteniendo también el ángulo de ataque óptimo $\left(\alpha_{\mathrm{op}}\right)$. Después del análisis de diferentes perfiles se opto por el perfil
Windtur 1, Figura 9, que resulto de la combinación entre el perfil SG 6043 y un NACA 6409.

Determinación del tamaño del rotor. El tamaño del rotor determina la cantidad de energía del viento que es manipulada. Aquí resulta importante realizar un diseño preliminar del rotor asumiendo una eficiencia global de entre $25 \%$ y $35 \%$ para rotores pequeños de paso fijo, o de $35 \%$ a $45 \%$ para rotores grandes de paso variable y calculando así la potencia de salida en relación a los requerimientos energéticos que exista. En este caso, la investigación determino un radio de 3 metros como base para el tamaño del rotor, según los requerimientos energéticos analizados.

Velocidades de operación. Cuando se conozca de forma aproximada la potencia de salida obtenida según el análisis anterior, se deben determinar las velocidades de operación, las cuales derivan de las condiciones eólicas existentes en los lugares para los cuales se esta diseñando el rotor. Una buena forma de aproximarse a estas velocidades resulta encontrando la velocidad del viento a la cual el viento posee su potencia promedio (mediante graficas de distribución de velocidad y potencia), y las velocidades de viento extremas promedio, garantizando que el diseño opere eficientemente la mayor parte del tiempo. Según los lugares analizados, se obtuvo una velocidad de viento mínima de $2.33 \mathrm{~m} / \mathrm{s}$, una velocidad máxima de $7.2 \mathrm{~m} / \mathrm{s}$ y una velocidad de diseño de $5 \mathrm{~m} / \mathrm{s}$.

\section{PROCEDIMIENTO DE DISEÑO}

Proceso teórico. Entre los diversos métodos existentes para el cálculo del rendimiento para un rotor de aerogenerador, se trabajó como base la teoría del momentum en el álabe (BEM), la cual permite establece una relación entre las fuerzas axiales y tangenciales existente en los álabes del aerogenerador (Fax y Ftan), y las existentes en una corriente fluida libre (Fax'y Ftan) [3].

$$
\begin{aligned}
& F a x=[(L i * \cos (\text { aoptimo }))+(D a * \operatorname{sen}(\text { aoptimo })]
\end{aligned}
$$

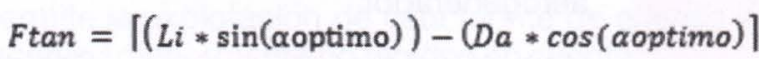

$$
\begin{aligned}
& \text { Fax }^{\prime}=\left(2 \pi * i *\left(\frac{L}{I}\right)\right) u((1-a) 2 a * u) \\
& \text { Ftan }^{\prime}=\left(2 \pi * i *\left(\frac{L}{I}\right)\right) u\left((1-a) 2 a^{\prime} * \omega * i\right)
\end{aligned}
$$

Dichas ecuaciones deben ser resueltas de tal forma que Fax = Fax' y Ftan = Ftan', para lo cual se requiere de un proceso iterativo probando diferentes valores de a ya'.

Resulta muy importante mencionar que este proceso iterativo debe realizarse en diversas estaciones de

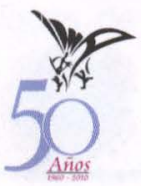




\section{álabe, para obtener así los factores de corrección de velocidad a través de todo el rotor.}

Este método, sin embargo, posee inconvenientes debido a que no tiene en cuenta el cambio de circulación a través del álabe, por lo que aquí resulta importante incluir la teoría de la circulación en el cálculo del rendimiento del rotor del aerogenerador.

Una adaptación de la ecuación común para obtener la circulación en cualquier estación de un ala de una aeronave utiliza reemplaza la velocidad del viento, por una velocidad relativa por estación $\mathrm{Vr}$, la cual es obtenida teniendo en cuenta la velocidad absoluta del viento y los factores de corrección a y a por estación.

$$
\Gamma(\phi)=4 * 2 l * \int_{0}^{l} F(V r l)\left(A_{1} \sin \phi \ldots \ldots .+A_{2 n-1} \sin \phi\right) d V r l
$$

Sin embargo, esta función no permite describir los posibles cambios de geometría del perfil y ángulos de ataque locales, por lo cual es propuesto aquí el siguiente método, el cual determina que la circulación total en el álabe representa una suma de funciones de circulación independientes y locales para cada estación del álabe.

$$
\Gamma=\sum_{\phi=0}^{\pi / 2} \Gamma(\phi)
$$

Aquí también resulta posible establecer el valor del factor de eficiencia de Oswald[4], el cual está determinado para cada estación y representado por una sumatoria de los coeficientes An locales.

$$
e=\frac{1}{1+\frac{3 A_{3}^{2}}{A_{1}^{2}}+\frac{5 A_{5}^{2}}{A_{1}^{2}}+\ldots \ldots .+\frac{(2 n-1) A_{2 n-1}{ }^{2}}{A_{1}^{2}}}
$$

Es así entonces que al realizar el proceso mostrado anteriormente para cada estación del álabe, y a diferentes velocidades de viento, puede calcularse el rendimiento del rotor para un aerogenerador.

Herramienta computarizada. Debido a la gran cantidad de cálculos requeridos por el modelo teórico, se realizó una programación de las diversas funciones relacionadas en MATLAB, y utilizando GUI (modo gráfico) para realizar comparaciones entre los resultados obtenidos.

Determinación del diseño. Para lograr un diseño optimo en la investigación, se procedió a utilizar la herramienta mencionada anteriormente para probar diferentes configuraciones geométricas y de rendimiento. Los resultados del diseño pueden observarse al final del documento, figuras 1,2 , y 13, Tabla 1.

\section{ELEMENTOS DE DISEÑO}

Algunos de los aspectos que intervienen en el rendimiento del rotor son los siguientes parámetros geométricos que fueron analizados mediante la herramienta computarizada mencionada anteriormente:

Efecto del aspect ratio (AR): En donde la potencia aumenta al aumentar el aspect ratio, esto a expensas de un aumento en la velocidad de arranque. Sin embargo un aumento muy grande del aspect ratio sin aumentar el TSR genera una disminución en la potencia, figuras 3 y 4.

Efecto del taperado $(\lambda)$ : La disminución del taperado contribuye al aumento del factor de eficiencia de Oswald y en general de las propiedades aerodinámicas del álabe, generando así un aumento en la potencia Además influye en la solidez ya que a mayor solidez genera un mayor obstáculo al flujo del viento y por lo tanto aumenta el valor del coeficiente "a", disminuyendo la energía presente en el viento y así la potencia, figuras 5 y 6 .

Efecto del tip speed ratio (TSR): La variación del TSR de diseño afecta la potencia, la velocidad de arranque debido al cambio en el twist, figura 7. Un aumento en el TSR de diseño, en conjunto con uno en el aspect ratio, aumentará la potencia de salida, a expensas de la velocidad de arranque. Resulta importante mencionar que cada rotor con un aspect ratio determinado, posee solo un valor de TSR óptimo. Como consecuencia directa de esto, el TSR de diseño afectara directamente el twist del álabe figura 8 , en donde un álabe con mayor TSR de diseño tendrá un ángulo de ataque absoluto con respecto a la horizontal.

Efectos del generador eléctrico: Dependiendo de la potencia del generador se presenta una variación en el cogging torque, por lo que un generador más potente requiere un mayor torque para arrancar, lo que trae como consecuencia una mayor velocidad de arranque. Asimismo, un generador con menor potencia no podrá producir una mayor cantidad de energía a velocidades de viento por encima de su velocidad de máxima potencia, por lo cual tendrá una velocidad de máxima potencia menor a la que posea un aerogenerador con un generador eléctrico de mayor potencia.

Efecto del número de álabes: El número de álabes a utilizar tiene diversos efectos en el desempeño del aerogenerador. Aunque un mayor número de álabes teóricamente aumenta en pequeña medida la eficiencia global, su construcción resulta más costosa y más complicada, debido a que los álabes deben tener aspect ratios muy elevados, donde una disminución exagerada de la cuerda local afecta negativamente el número de Reynolds y en consecuencia el rendimiento. Asimismo los rotores con números de álabes pares pueden presentar algunos problemas relacionados con vibraciones. Según los resultados de esta investigación, se recomienda utilizar rotores de 3 y 5 álabes, dependiendo de las condiciones de diseño y el número de Reynolds. 
Comparación de resultados teóricos y los experimentales

La investigación realizó una comparación de los resultados arrojados por la herramienta calculando aerogeneradores reales y un modelo a escala que fue probado en un túnel de viento de baja velocidad de la Universidad de San Buenaventura, Bogotá - Colombia.

Comparación con aerogeneradores reales. Aquí se realizó una simulación de las características geométricas aproximadas de los aerogeneradores Vestas V52 y Westwind $5.5 \mathrm{~kW}$, dejando que la propia herramienta determinara el twist óptimo para cada caso. En términos generales, los resultados obtenidos muestran una elevada correlación entre los datos provenientes de la herramienta y del fabricante, figuras 10 y 11.

Comparación con modelo experimental. En este caso se procedió a realizar un modelo diseñado por la herramienta y probarlo en el túnel de viento a números de Reynolds de entre 5000 y 35.000. Para modelar el factor de reducción de la velocidad axial "a", se procedió a medir la velocidad del viento con el modelo estático y con el modelo girando, factor que fue modelado luego en la herramienta computarizada. Los resultados obtenidos, figura 12, no muestran una clara relación entre los datos esperados por la herramienta y los datos medidos experimentalmente, posiblemente debido a otros factores difícilmente manejables como la perdida de potencia en el generador eléctrico DC utilizado y a la dificultad de modelar la expansión de la corriente fluida antes del rotor (para lo cual se utilizó el factor "a" mencionado anteriormente).

Como consecuencia del experimento, se observa que el túnel de viento debe ser utilizado con mucha precaución en el moldeamiento de aerogeneradores, además aquí es recomendable adoptar algunos otros modelos de prueba, como la utilización de una "cascada de álabes", utilizado comúnmente para pruebas de modelos giratorios en un túnel de viento[5].

\section{Uso de winglets como método de optimización de rendimiento}

Aunque el uso de winglets se ha vuelto popular en aeronaves, el uso de estos dispositivos en aerogeneradores se presenta en muy pocas ocasiones. Investigaciones recientes han probado que el uso adecuado de winglets puede aumentar la producción de energía de un aerogenerador en más de un $1.6 \%[6]$. Debido a la ausencia de metodologías para el diseño de winglets para aerogeneradores, se procedió a utilizar como referencia, una metodología de diseño de winglets para planeadores [7][8], siendo las alas de los planeadores en muchos aspectos muy parecidas a los álabes de los aerogeneradores.

\section{Análisis estructural general}

Cargas que actúan sobre el rotor. Las cargas que actúan sobre el rotor durante su operación deben mantenerse dentro de los límites de fatiga y pandeo ya que en el álabe aparecen esfuerzos de tracción y compresión que generan la fatiga.

Parámetros de diseño estructural. Los parámetros de diseño estructural se basan principalmente en estándares internacionales de diseño, entre los cuales se pueden destacar "Regulation for the Certification of Wind Energy Conversion Systems" de Germanischer-Lloydn, el estándar Danés DS472 y el estándar de requerimientos de seguridad para sistemas generadores de turbinas eólicas propuesto por la Comisión Internacional de Electrotecnia (IEC 61400-1)

Determinación de las características estructurales. Para determinar el peso del álabe se utiliza una estructura tipo "sándwich" de espuma de poliuretano con laminado de fibra de vidrio (Tabla 2), como estructura de referencia y se determinan las cargas máximas por estación a las que está expuesto el álabe aplicando así los factores de seguridad requeridos según el caso. Estos factores provienen de los estándares. Para rotores grandes aquí es posible utilizar un proceso alterno basado en ecuaciones empíricas[9].

Procedimiento de análisis. Para realizar el análisis estructural se tomó como punto de partida las cargas axiales en el rotor a diferentes velocidades de viento. Estas cargas axiales Fax determinadas por la iteración mostrada en el proceso teórico, conjunto con el estudio de las cargas por estación y el estudio del peso del álabe, permiten obtener las cargas dinámicas en el álabe, las cuales deben ser analizadas según los estándares de diseño. Esto trae como resultado final la magnitud de las cargas tanto estáticas como dinámicas en el rotor (Tabla 3), lo que permite culminar el diseño general del álabe del rotor (Tabla 4)

\section{Notas Finales}

El diseño de un rotor para un aerogenerador de bajas velocidades representa una idea lógica debido a que permite la explotación de esta fuente de energía en lugares anteriormente no tenidos en cuenta.

El modelo teórico utilizado para realizar este diseño resulta válido, debido a que se encuentra una gran concordancia entre los datos provenientes del análisis de aerogeneradores reales, con datos de los mismos fabricantes.

Aunque existen algunas debilidades concernientes al diseño de winglets y la forma en la cual la teoría de la circulación modela las puntas de los álabes con aspect ratios elevados y taperados cercanos a cero, el método propuesto y su validación demuestra que el diseño realizado es el más apropiado para las variables tenidas en cuenta y las condiciones de operación dadas.

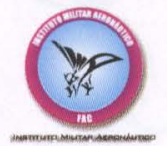




\section{REFERENCIAS}

[1] Perfiles SG. Diseñados por Michael Seling (S) y Phillipe Giguerre (G). Universidad de Illinois.

[2] Munson, Brucer, Young, Donald y Okiishi, Theodore. Fundamentos de mecánica de fluidos. Limusa. 2003.

[3] Corten, Gustave. Flow separation on wind turbine blades. 2001

[4] Bertin, Jhon and SMITH, Michael L. Aerodynamics for Engineers. Prentice Hall. 1998.

[5] Saravanamutto, Rogers and Cohen "Gas Turbine Theory" Pearson.2001

[6] Johansen and Sørensen. Aerodynamic investigation of winglets on wind turbine blades using CFD. RIS $\varnothing$ National Library. 2006 www.risoe.dk/rispubl/VEA/veapdf/risr-1543.pdf

[7] Maughmer, Mark. The design and testing of a winglet airfoil for low-speed aircraft.

www.mandhsoaring.com/articles/Winglet_ Testing.pdf

[8] Maughmer, Mark. The design of winglets for high-performance sailplanes.

www.mandhsoaring.com/articles/Winglet_ Design.pdf

[9] Bir and Migliore. Preliminary structural design of composite blades for two- and three-blade rotors

\section{LISTA DE TABLAS}

Tabla 1. Datos técnicos ${ }^{\dagger}$ de un aerogenerador que utiliza el rotor diseñado.

\section{DATOS TÉCNICOS AEROGENERADOR}

Número de álabes 3

Diámetro del rotor $6 \mathrm{~m}$

Velocidad de arranque $\quad 2.33 \mathrm{~m} / \mathrm{s}$

Max. Potencia

3 kW @ $7.66 \mathrm{~m} / \mathrm{s}$

Potencia a $5 \mathrm{~m} / \mathrm{s}$

$815 \mathrm{~W}$

TSR @ $5 \mathrm{~m} / \mathrm{s}$

Rpm del rotor@ 5 m/s

84.4

Coef. de potencia @ 5 m/s 0.37

Área de la Veleta guía

$3 m^{2}$

$†$ Datos técnicos de rendimiento referenciados al nivel

del mar, atmosfera estándar, sin winglets, y una

eficiencia electromecánica de 0.9 .

Tabla 2. Materiales propuestos para el álabe

\begin{tabular}{|c|c|c|c|}
\hline Material & $\begin{array}{c}\text { Densidad } \\
\mathrm{Kg} / \mathrm{cm}^{3}\end{array}$ & $\begin{array}{c}\text { Esfuerzo } \\
\text { último } \\
\text { (longitudinal) }\end{array}$ & $\begin{array}{c}\mathrm{E} \\
\text { (longitudinal) }\end{array}$ \\
\hline $\begin{array}{c}\text { Fibra de } \\
\text { vidrio S }\end{array}$ & 2.49 & $3500 \mathrm{Mpa}$ & $71 \mathrm{GPa}$ \\
\hline Epoxi & 1.54 & $60 \mathrm{Mpa}$ & $3.5 \mathrm{Gpa}$ \\
\hline $\begin{array}{c}\text { Espuma de } \\
\text { Poliuretano }\end{array}$ & 0.06 & $2.06 \mathrm{MPa}$ & $41.3 \mathrm{MPa}$ \\
\hline
\end{tabular}

Tabla 3. Cargas y momentos de diseño para rotor álabe

\begin{tabular}{|c|c|}
\hline CARGA & RESULTADO \\
\hline Axial máxima & 109.2 Newton \\
\hline Tangencial máxima & 27.6 Newton \\
\hline Drag álabe & 113.4 Newton \\
\hline Drag rotor & 252.43 Newton \\
\hline Drag álabe estático & 33.70 Newton \\
\hline Drag rotor estático & 74.93 Newton \\
\hline Fuerza centrífuga & 73.31 Newton \\
\hline Momento flector álabe & 76.44 Newton metro \\
\hline $\begin{array}{c}\text { Momento flector álabe } \\
\text { estático }\end{array}$ & 22.72 Newton metro \\
\hline $\begin{array}{c}\text { Momento torsion rotor } \\
\text { Momento torsion álabe }\end{array}$ & 3.84 Newton metro \\
\hline $\begin{array}{c}\text { Carga ultima a tension } \\
\text { del álabe }\end{array}$ & 102.74 Newton \\
\hline
\end{tabular}

Tabla 4. Datos técnicos del rotor

Número de álabes

Diámetro del rotor

Tipo de rotor

Perfil aerodinámico

del álabe

Perfil aerodinámico

de los winglets

Aspect ratio del álabe

Taperado del álabe

Ángulo de flechamiento del álabe @ 25\% de la cuerda

Longitud de los winglets

Taperado de los winglets

Ángulo de flechamiento de los winglets @ 25\%

de la cuerda

Peso de cada álabe

\section{3}

$6 \mathrm{~m}$

Paso fijo

Windtur 1

PSU 94-097

30

0 grados

0.12 metros

0.5

20 grados

$1.756 \mathrm{~kg}$ 


\section{REFERENCIAS \\ [1] Perfiles SG. Diseñados por Michael Seling (S) y Phillipe Giguerre (G). Universidad de Illinois. \\ [2] Munson, Brucer, Young, Donald y Okiishi, Theodore. Fundamentos de mecánica de fluidos. Limusa. 2003. \\ [3] Corten, Gustave. Flow separation on wind turbine blades. 2001 \\ [4] Bertin, Jhon and SMITH, Michael L. Aerodynamics for Engineers. Prentice Hall. 1998. \\ [5] Saravanamutto, Rogers and Cohen "Gas Turbine Theory" Pearson.2001 \\ [6] Johansen and Sørensen. Aerodynamic investigation of winglets on wind turbine blades using CFD. RIS $\varnothing$ National Library. 2006 www.risoe.dk/rispubl/VEA/veapdf/ris- r-1543.pdf \\ [7] Maughmer, Mark. The design and testing of a winglet airfoil for low-speed aircraft. \\ www.mandhsoaring.com/articles/Winglet_ Testing.pdf \\ [8] Maughmer, Mark. The design of winglets for high-performance sailplanes. \\ www.mandhsoaring.com/articles/Winglet_ Design.pdf \\ [9] Bir and Migliore. Preliminary structural design of composite blades for two- and three-blade rotors}

\section{LISTA DE TABLAS}

Tabla 1. Datos técnicos ${ }^{\dagger}$ de un aerogenerador que utiliza el rotor diseñado.

\section{DATOS TÉCNICOS AEROGENERADOR}

Número de álabes 3

Diámetro del rotor $6 \mathrm{~m}$

Velocidad de arranque $2.33 \mathrm{~m} / \mathrm{s}$

Max. Potencia $3 \mathrm{~kW} @ 7.66 \mathrm{~m} / \mathrm{s}$

Potencia a $5 \mathrm{~m} / \mathrm{s}$ $815 \mathrm{~W}$

TSR @ $5 \mathrm{~m} / \mathrm{s}$

Rpm del rotor@ $5 \mathrm{~m} / \mathrm{s}$

Coef. de potencia @ 5 m/s 0.37

Área de la Veleta guía

$3 m^{2}$
Tabla 2. Materiales propuestos para el álabe

\begin{tabular}{|c|c|c|c|}
\hline Material & $\begin{array}{c}\text { Densidad } \\
\mathrm{Kg} / \mathrm{cm}^{3}\end{array}$ & $\begin{array}{c}\text { Esfuerzo } \\
\text { último } \\
\text { (longitudinal) }\end{array}$ & $\begin{array}{c}\mathrm{E} \\
\text { (longitudinal) }\end{array}$ \\
\hline $\begin{array}{l}\text { Fibra de } \\
\text { vidrio S }\end{array}$ & 2.49 & $3500 \mathrm{Mpa}$ & $71 \mathrm{GPa}$ \\
\hline Epoxi & 1.54 & $60 \mathrm{Mpa}$ & $3.5 \mathrm{Gpa}$ \\
\hline $\begin{array}{l}\text { Espuma de } \\
\text { Poliuretano }\end{array}$ & 0.06 & $2.06 \mathrm{MPa}$ & $41.3 \mathrm{MPa}$ \\
\hline
\end{tabular}

Tabla 3. Cargas y momentos de diseño para rotor álabe

\begin{tabular}{|c|c|}
\hline CARGA & RESULTADO \\
\hline Axial máxima & 109.2 Newton \\
\hline Tangencial máxima & 27.6 Newton \\
\hline Drag álabe & 113.4 Newton \\
\hline Drag rotor & 252.43 Newton \\
\hline Drag álabe estático & 33.70 Newton \\
\hline Drag rotor estático & 74.93 Newton \\
\hline Fuerza centrífuga & 73.31 Newton \\
\hline Momento flector álabe & 76.44 Newton metro \\
\hline $\begin{array}{c}\text { Momento flector álabe } \\
\text { estático }\end{array}$ & 22.72 Newton metro \\
\hline $\begin{array}{c}\text { Momento torsion rotor } \\
\text { Momento torsion álabe }\end{array}$ & 11.54 Newton metro \\
\hline $\begin{array}{c}\text { Carga ultima a tension } \\
\text { del álabe }\end{array}$ & 102.74 Newton \\
\hline
\end{tabular}

\section{Tabla 4. Datos técnicos del rotor}

Número de álabes

Diámetro del rotor

Tipo de rotor

Perfil aerodinámico

del álabe

Perfil aerodinámico

de los winglets

Aspect ratio del álabe

Taperado del álabe

Ángulo de flechamiento del álabe @ 25\% de la cuerda

Longitud de los winglets

Taperado de los winglets

Ángulo de flechamiento de los winglets @ 25\% de la cuerda

Peso de cada álabe

\section{3}

$6 \mathrm{~m}$

Paso fijo

Windtur 1

PSU 94-097

0 grados

0.12 metros

0.5

20 grados

$1.756 \mathrm{~kg}$ 


\section{LISTA DE FIGURAS}

Figura 1. Apariencia del rotor de un aerogenerador de bajas velocidades.

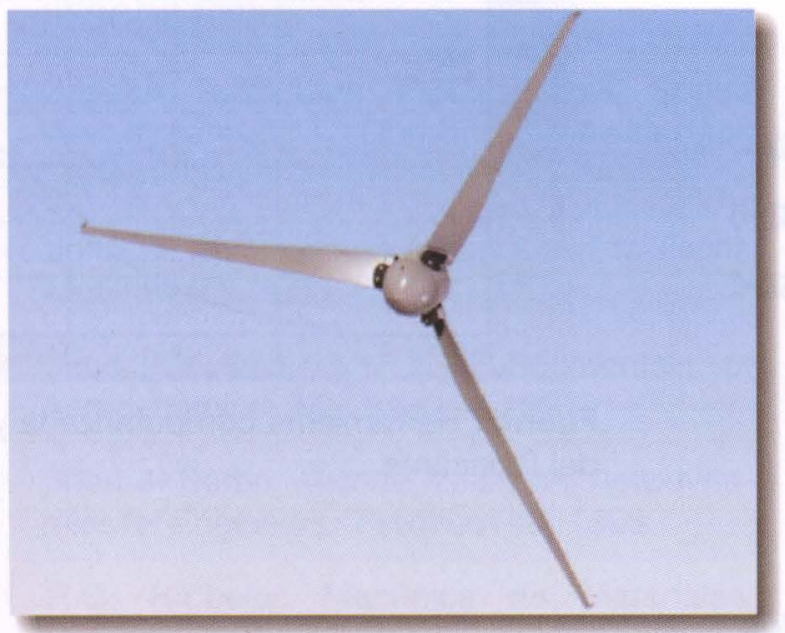

Figura 2. Detalle del álabe de un rotor para un aerogenerador de bajas velocidades.

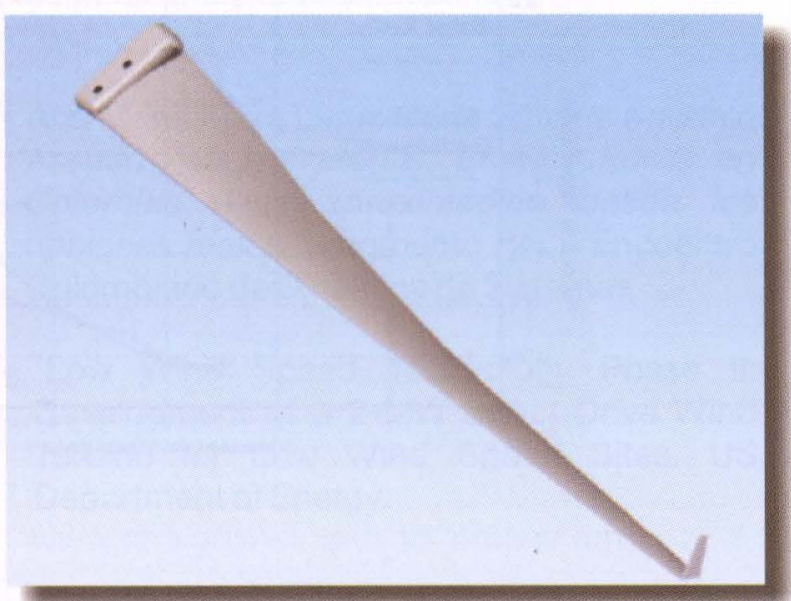

Figura 3. Variación de la potencia producida con diferentes valores de AR en una turbina eólica con un radio de 3 metros, TSR de 3 y $\lambda$ de 0.2 .

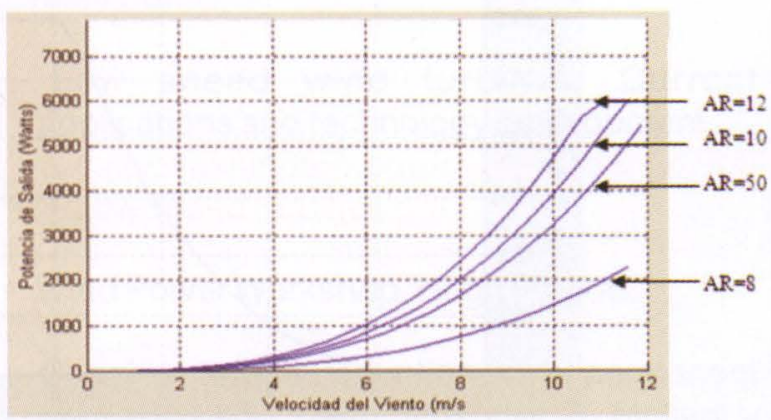

Figura 4. Variación en la velocidad de arranque debido al AR en una turbina eólica con un radio de 3 metros, TSR de 3 y $\lambda$ de 0.2 .

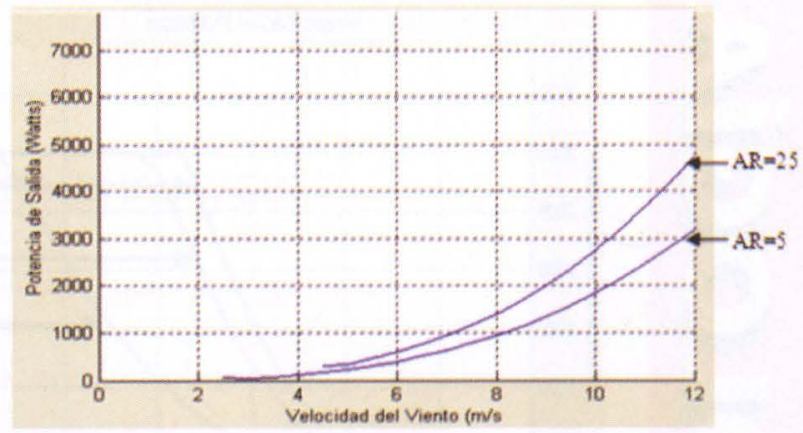

Fuente: Herramienta computarizada

Figura 5. Variación de la potencia debido a los diferentes valores de $\lambda$ en una turbina eólica de 3kw, 3 metros de radio, TSR de 4 y AR de 25.

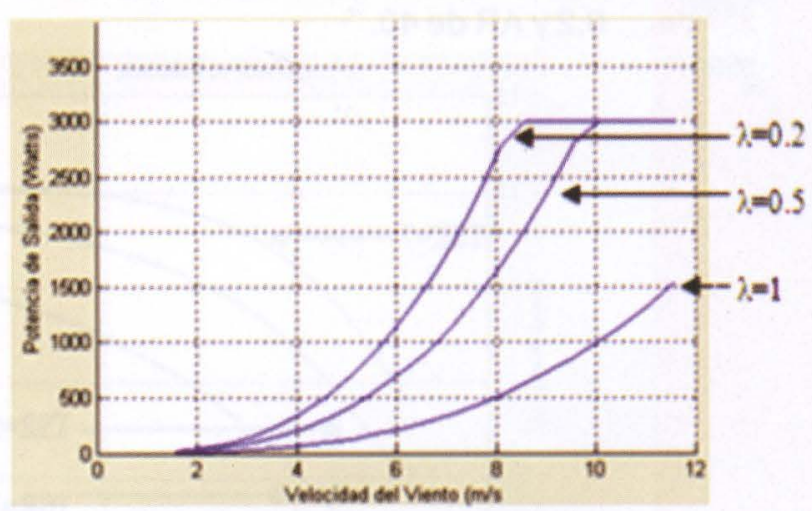

Fuente: Herramienta computarizada

Figura 6. Variación del $\mathrm{CL}$ en diferentes estaciones debido a los diferentes valores de $\lambda$ en una turbina eólica de $3 \mathrm{kw}, 3$ metros de radio, TSR de 4 y AR de 25.

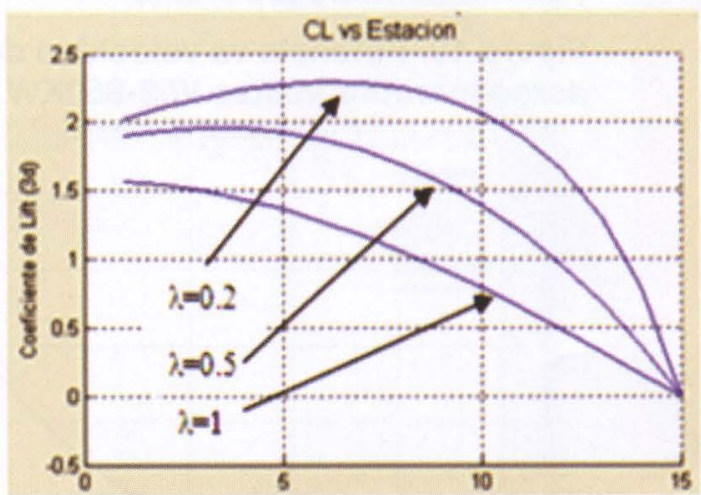

Fuente: Herramienta computarizada

Fuente: Herramienta computarizada

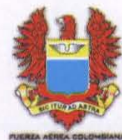




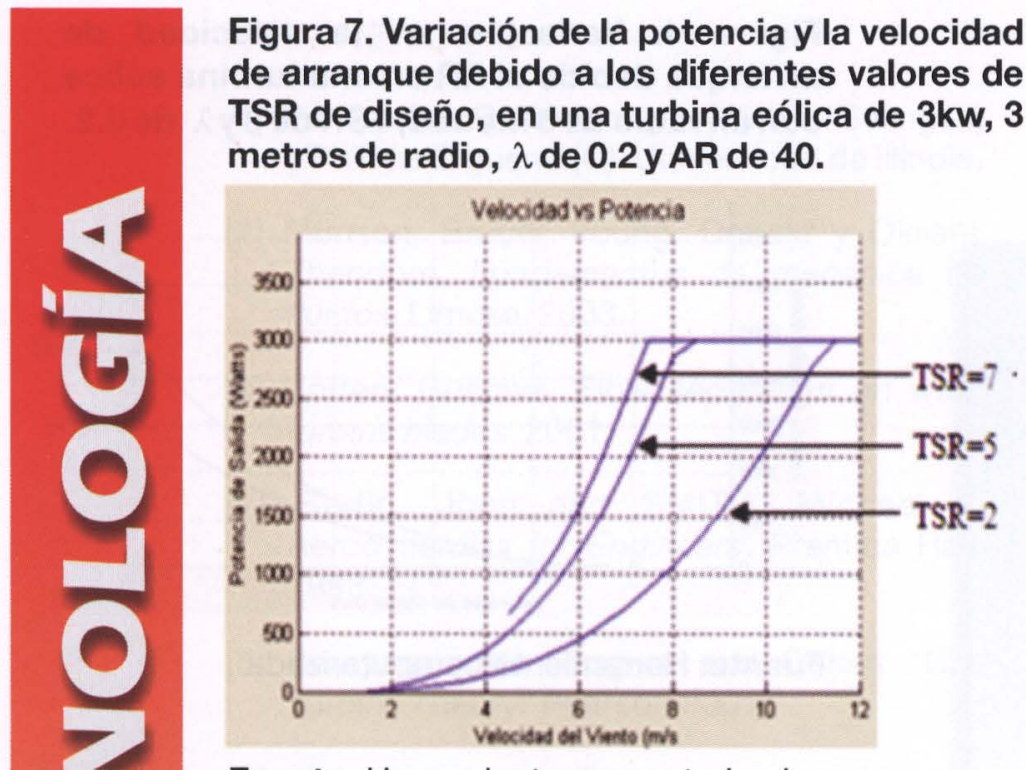

Fuente: Herramienta computarizada

Figura 8. Variación del twist debido a los diferentes valores de TSR de diseño, en una turbina eólica de $3 \mathrm{kw}, 3$ metros de radio, $\lambda$ de 0.2 y AR de 40.

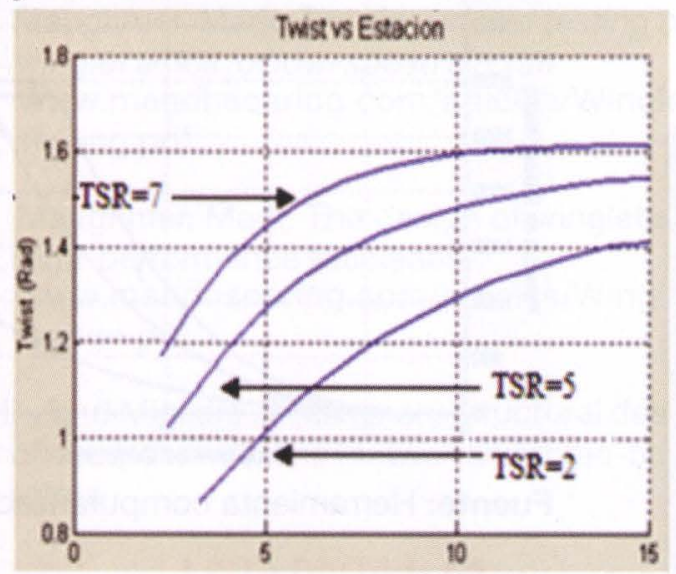

Fuente: Herramienta computarizada

Figura 9. Perfil Windtur1

Fuente: Software profili 2.16

Figura 10. Potencia vs velocidad del aerogenerador Vestas V52-850KW.

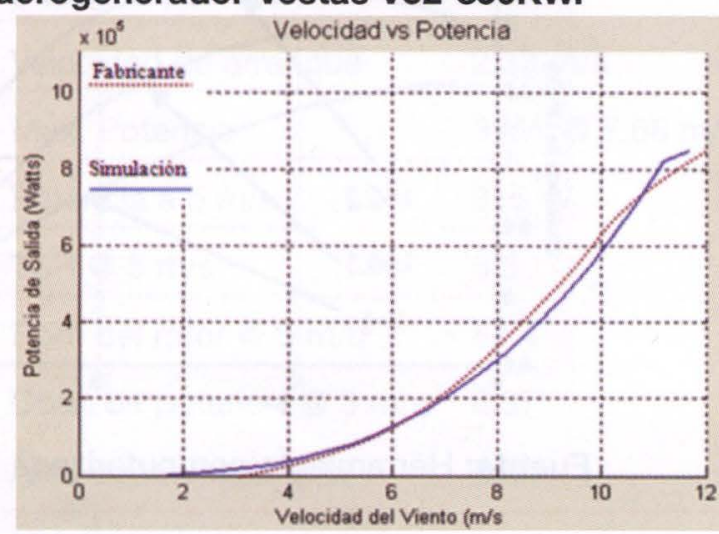

Fuente: Herramienta computarizada y datos

del fabricante
Figura 11. Potencia vs velocidad del aerogenerador Westwind $5.5 \mathrm{Kw}$.

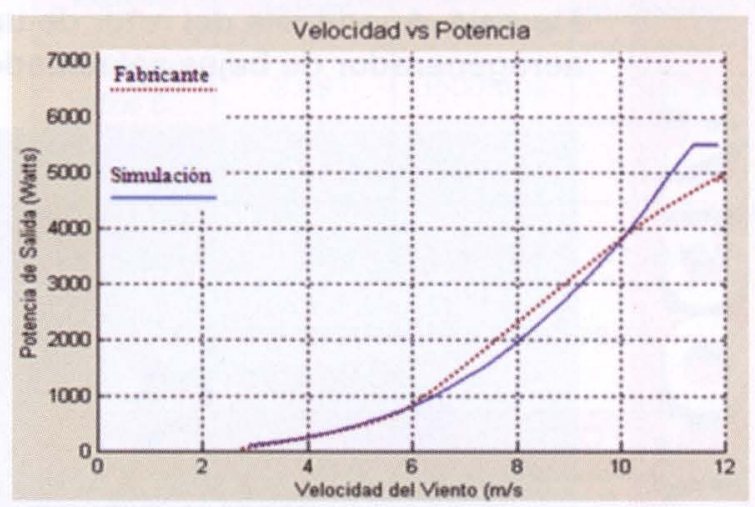

Fuente: Herramienta computarizada y datos del fabricante

Figura 12. Potencia vs. Velocidad del modelo de aerogenerador y su simulación en la herramienta.

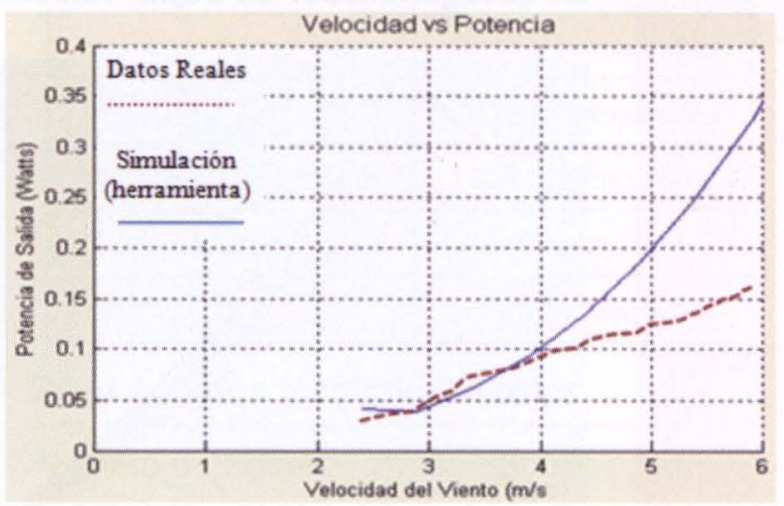

Figura 13. Potencia vs. velocidad del aerogenerador que usa el rotor diseñado.

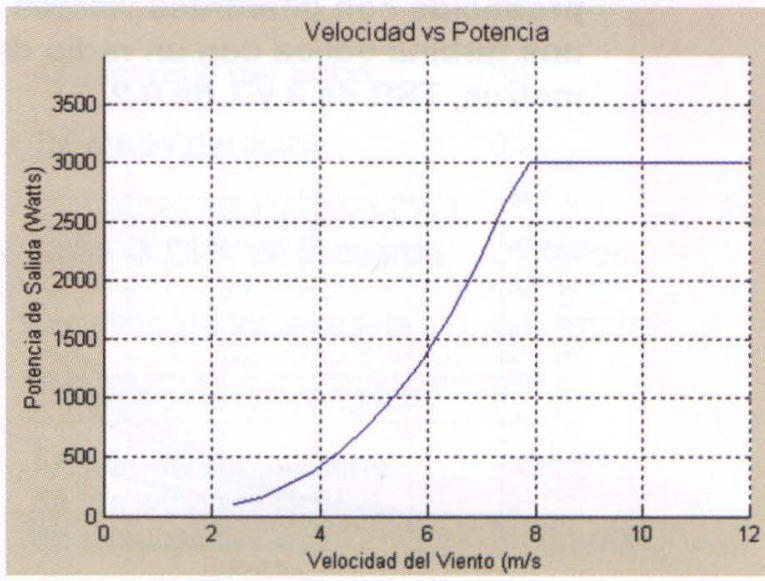

Fuente: Herramienta computarizada 


\section{REFERENCIAS}

- Ernesto Sánchez Triana, Carlos Silva Fernández. Eficiencia energética, producción limpia para un desarrollo sustentable. FESCOL 1996

- Joseph Katz-Allen Plotkin. Low Speed Aerodynamics. Cambridge Aerospace Series 2001.

- John D. Anderson Jr. Introduction to Flight. McGraw Hill. 2000

- Jhon D. Anderson Jr. Fundamentals of Aerodynamics. McGraw Hill. 1991

- Jhon J. Bertin, Michael L. Smith. Aerodynamics for Engineers. Prentice Hall. 1998.

- R.C. Hibbeler. Mecánica de Materiales. Pearson. 2001

\section{Documentos PDF.}

- Sistema Ambiental de Colombia. IDEAM.

www.ideam.gov.co/publica/medioamb/cap3i.pdf

- Ana María Mora Luna, Carla Susana Agudelo Assuad, Isaac Dyner R. Energia Eólica en Colombia: Una aproximación desde las opciones reales. Fragmento del II Encuentro Colombiano de Dinámica de Sistemas

- Low Wind Speed Technology Phase II: Development of a 2-MW Direct-Drive Wind Turbine for Low Wind Speed Sites. US Department of Energy.

www.nrel.gov/wind/wind_project.html

- Burton, Sharpe, Jenkins, Bossanyi. Wind Energy Handbook. WILEY. 2001

- Wind Technology: The Facts. European Wind Energy Association.

www.ewea.org/fileadmin/ewea_documents/ documents/publications/WETF/Facts_Sum mary.pdf

- Low speed wind turbines: Current applications and technology development. faculty.washington.edu/malte/seminar/Sp0 4/LowSpeedTurbines.pdf

- Wind Power Workshop. Hugh Piggott.

- Cogging torque reduction in a permanent magnet wind turbine generator, E. Muljadi y J. Green.
- Jeppe Johansen y Niels N. Sørensen. Aerodynamic investigation of Winglets on wind turbine blades using CFD. Ris $\varnothing$ National Library. Febrero del 2006

www.risoe.dk/rispubl/VEA/veapdf/ris-r1543.pdf

\section{PÁGINAS WEB}

\section{- www.windpower.org}

- www.mf-ct.upc.es/JMBergada/mf/practicas/ Flujo exteno-pract11.doc

- www.termica.webhop.info/

- www.exa.unicen.edu.ar/fluidos/pdf/delnero.pdf

- www.imefen.uni.edu.pe/mfluidos/13va\%20clase.pdf 